Automobilindustrie

\section{Nikola versus Tesla}

Elektroautopionier Elon Musk war von dem kroatischen Physiker Nikola Tesla so angetan, dass er sein Start-up Tesla nannte. Tesla ist Innovationsführer bei batterie-elektrischen Autos. Die Tesla-Aktie wurde am 9. Juni 2020 an der Nasdaq mit 833 Euro bewertet. Das war übrigens kurz nachdem Nikola Motors an die Börse ging. Natürlich soll der Name Nikola Motors die Assoziation mit Tesla herstellen. Geplant ist, die Welt mit Brennstoffzellen-Autos, genauer gesagt Pick-ups und Lastwagen, zu verändern. Sowohl Tesla als auch Nikola Motors produzieren Elektroautos. Bei Tesla kommt er Strom allerdings aus der Steckdose und bei Nikola aus einer kleinen Chemiefabrik - die Brennstoffzellen-Stacks - im Auto, die aus Wasserstoff Strom erzeugt. Nikola hat noch kein Auto gebaut und auch keine Fabrik. Bisher ist es eher eine Blaupause mit furiosem Börsenstart. Mittlerweile ist der Börsenkurs vom 9. Juni 2020 mit 74 Euro auf 26 Euro Ende Juli abgesackt. Ende Juli waren Tesla-Aktien übrigens 1.219 Euro wert, knapp ein Drittel mehr als zum Börsenstart von Nikola. Ist also grüner Wasserstoff beim Autoantrieb ein Flop?

Wasserstoff ist ein farb- und geruchloses Gas. Für Bundesforschungsministerin Anja Karliczek ist er grün und „das Erdöl von morgen“. Für sie wird „mit der Nationalen Wasserstoffstrategie Deutschland zu einem globalen Vorreiter“. Natürlich gab es auch eine „Nationale Elektromobilitätsstrategie" und Wirtschaftsminister Peter Altmaier hat Pläne für deutsche Batterie-Champions. Die Bundesregierung subventioniert mit 1 Mrd. Euro die Batteriezellfertigung. Batterie ist so etwas Ähnliches wie „das Erdöl von morgen“. Brauchen wir also beides? Wie passt das alles ins Bild eines Technologiestandorts Deutschland? Mit der Energiewende wurde die „grundlegend neue Energieversorgung Deutschlands“ ausgerufen. Zwischendurch werden die Abhängigkeit von Medizinprodukten aus China ausgehebelt, die Maskenproduktion in Deutschland aufgebaut und natürlich nationale Digitalisierungs-Champions 2.0, 3.0, 4.0 auf den Weg gebracht - Deutschland als Silicon-Germany. Man könnte den Eindruck gewinnen, die Minister verbreiten jede Woche eine neue Vision. Was fehlt, ist die Langfristigkeit, die Strategie. Deutschland hatte früher eine Spitzenstellung in der Kernenergie. Deutschland hatte auch vor zig

(C) Der/die Autor(en) 2020. Open Access: Dieser Artikel wird unter der Creative Commons Namensnennung 4.0 International Lizenz (https:// creativecommons.org/licenses/by/4.0/deed.de) veröffentlicht.

Open Access wird durch die ZBW - Leibniz-Informationszentrum Wirtschaft gefördert.
Jahren eine passable Position in der Elektrochemie, Konrad Zuse baute den ersten mechanischen Rechner, und, und, und. Unsere Forschungs- und Wirtschaftspolitik ist schlagzeilengetrieben und nicht strategisch. Technologievorteile gewinnt man durch strategisches Handeln und nicht dadurch, dass man hin und her springt.

Aber kommen wir zu Nikola Tesla zurück und der Frage, welche Speichertechnik für Sonnen- oder Windenergie für Elektroautos die effizienteste ist. Das niederländische Forschungsinstitut TNO etwa kommt zu dem Ergebnis, dass bei $100 \%$ erneuerbarem Energieeinsatz beim batterieelektrischen Auto nur $73 \%$ der Energie zum Vortrieb genutzt werden kann. Beim Transport und der Umwandlung von elektrischer Energie in mechanische Energie entsteht „Verlustleistung“. Transformiert man Windstrom in Wasserstoff und macht dann aus dem Wasserstoff über die Brennstoffzelle wieder elektrischen Strom, verbleiben gerade einmal $22 \%$ zum Vortrieb. Viel schlechter kann eine Energiebilanz nicht sein. Ist grüner Wasserstoff also das Erdöl von morgen? Beim Auto irrt Ministerin Karliczek.

Der große Vorteil des Wasserstoff-Brennstoffzellen-Systems liegt in der Tankgeschwindigkeit. Der Nachteil liegt im Preis der Brennstoffzelle und der teuren Tankinfrastruktur. Für Busse in einer Großstadt oder den Lkw-Verkehr von Ankara nach Stockholm wäre das eher vorstellbar. Entlang einer Hauptroute ist alle $300 \mathrm{~km}$ eine Tankstelle erforderlich. Aber auch hier ist das Rennen noch nicht ganz gelaufen. Batterietechnologien entwickeln sich weiter. Und für Stadtbusse ist Salzburg mit seiner Technik für Oberleitungsbusse vielleicht auch eine Alternative. Vielleicht schafft man es sogar, mehr Gütertransporte auf die Schiene zu bringen. Und nicht vergessen, Elon Musk arbeitet an seinem Semi-Truck. Man sollte inn nicht unterschätzen.

Zusammenfassend ist der grüne Wasserstoff im PkwMarkt schwer vorstellbar, im Nutzfahrzeugverkehr könnte er sinnvoll sein. Dass wir Speichertechnologien für Elektrizität brauchen, liegt auf der Hand. Ob grüner Wasserstoff wirklich das Erdöl von morgen ist oder Peter Altmaier morgen eine neue zündende Idee für die Nation hat, ist offen. Wenig verständlich ist, dass wir uns ein Sprechverbot für das Wort „Kernenergie“ auferlegt haben. Alle bisherigen Daten zeigen, dass Kernenergie - auch nach Harrisburg, Tschernobyl und Fukushima - deutlich weniger gefährlich ist als viele vermuten. Was Deutschland braucht, ist eine richtige Technologie-Strategie und keine hektischen Ministerpläne mit Platituden à la „Erdöl von morgen“. Nur langer Atem schafft Wettbewerbsvorteile.

Ferdinand Dudenhöffer CAR-Center Automotive Research, Duisburg Ferdi.Dudenhoeffer@car-future.com 